aus, entsprechend dem Wachstum der Sphagnumrasen in der vorhin angedeuteten Weise, unter allmählicher Verflachung zentrifugal ausgebreitet haben. Wegen ihrer Gestalt sind die Hochmoore gewöhnlich leicht zu entwässern. Indessen ist die uhrglasartige Aufwölbung der Oberfläche nicht immer vorhanden, und das Hochmoor gelegentlich der Gestalt nach ein schwieriger zu entwässerndes Fla c h m o or, d. h. ein Moor mit nahezu wagerechter Oberfläche.

Die ursprüngliche Vegetation der Hochmoore ist im gegenwärtigen Zeitalter ein geschlossener Sphagnumrasen, in dem andere Pflanzen nur spärlich eingestreut leben können. Sobald durch die Anlage von Torfgruben oder von Gräben die Entwässerung eingeleitet worden ist - bei kleineren Hochmooren kann auch eine Beseitigung des feuchthaltenden Waldes der Umgebung den gleichen Eirfolg haben -, so sterben die Sphagnen $a b$, und das Gelände bedeckt sich statt ihrer mit Heidesträuchern. Diesen Zustand zeigt gegenwärtig die überwiegende Mehrzahl der Hochmoore Deutschlands. Dabei hat sich eine von der Oberfläche nach unten fortschreitende Vermoderung des jüngern Sphagnumtorfs eingeleitet und zur Bildung einer mehr oder minder starken Verwitterungsrinde geführt. Der ältere Sphagnumtorf liefert einen wertvollen Brennstoff, während der jüngere zur Herstellung einer besonders geschätzten Torfstreu dient. -

Ein Rückblick auf die Entstehung der Moore lehrt, da $\beta$ es sich dabei allerdings in erster Reihe um botanische und geologische Erscheinungen handelt. Aber wie beide Wissenschaften sich anderweit die Chemie nutzbar gemacht haben, so wäre zu wünsohen, daß ein gleiches in noch größerm Umfange als bisher auch auf diesem Gebiete erfolgte. Insbesondere harrt die Erforschung des Vertorfungsvorgangs und der Diagenese der Humusstoffe, sowie der Erzeugnisse beider Prozesse noch einer eindringlichern chemischen Bearbeitung als bisher. Es ist zu erwarten, daß sich dadurch manche Fragen der Entstehungsgeschichte der Humusbildungen, des gegenwärtigen wie früherer Zeitalter, mit größerer Sicherheit werden beantworten lassen als zurzeit möglich ist.

\section{Über die Geschichte des Schwefelsäurekontaktprozesses II.} Von Dr. F. WINTELER. (Eingeg. d. 27.7. 1905.)

Die Darstellung von Schwefelsäure soll nach dem ein Jahr nach Wöhlers Versuchen aus- gestellten englischen Patent 731/1853, durch Uberleiten von Schwefelkiesröstgasen über Eisenoxyd in Form von Kiesabbränden geschehen. Die Abbrände befinden sich in einem Ofen oder Schacht bei dunkler Rotglut, die durch die Hitze der Röstgase selbst erzeugt wird, die Luft wird am Boden des Schachts zugelassen, um einen genügenden Sauerstoffüberschuß zu bekommen.

Die nunmehr in den nächsten Jahren folgenden Veröffentlichungen bieten nichts wesentlich Neues mehr. Da die englische Patentgesetzgebung eine Vorprüfung angemeldeter Erfindungen zum Patentschutz nicht kennt, so zeigt sich die Erscheinung, daß früher patentierte Erfindungsgedanken mehrmals nachpatentiert werden können.

Am 25./1. 1854 erhält $W$ illi a m Henry Thornthwaite das englische Patent 188 , worin nochmals die Verwendung von Platin und Oxyden unedler Metalle geschützt wird. Als Verbesserung in der Fabrikation von Schwefelsäure wird angegeben: ,Die Erfindung bezieht sich auf die Verwendung von einem katalysierenden Material, wie platinierter Asbest oder einer platinierten Substanz, welche von Schwefelsäure nicht verändert wird, von Chromsesquioxyd, Eisensesquioxyd, Mangansesquioxyd oder anderer Sesquioxyde zum Zwecke die Verbindung von Sauerstoffgas (aus gewöhnlicher Luft oder anderen Quellen herrührend) in Gegenwart von schwefliger Säure herbeizuführen oder zu unterstïtzen, und auf diese Weise Schwefelsäure zu erzeugen". Ich erreiche das in der Weise, indem ich Schwefelsäuregas mit der notwendigen Menge gewöhnlicher Luft gemischt über die katalysierende Substanz streichen lasse, welche vorher erhitzt wurde, oder indem ich die Gae von einem brennenden Gemisch von Schwefel und Salpeter wie sie gewöhnlich für die Fabrikation von Schwefelsäure gebraucht werden, über die erhitzte katalysierende Masse leite, wodurch eine beträchtliche Ersparnis von Salpeter erreicht wird.

Das kurz darauf erteilte englische Patent 982 von demselben Jahre an Alf r. Truemann ausgestellt, hat den Titel: Verbesserungen in der Darstellung von Schwefelsäure beim Rösten von Kupfererzen, beim Verbrennen von Schwefel und Eisenkiesen.

,Die Erfindung besteht darin, daß man die schweflige Säure, welche beim Rösten von Kupfererzen usw. erhalten wird, gemischt mit Sauerstoff oder Luft oder sonstiger Materie, welche Sauerstoff enthält, über erhitztes Platin, Eisenoxyd oder andere Substanz streichen läßt, welche in erhitztem Zustande die Eigenschaft hat, Sauerstoff mit schwefliger Säure zu verbinden und Schwefelsäure zu erzeugen". Die Erfindung besteht ebenfalls darin, Platin, Eisenoxyd oder andere Substanz (welche in erhitztem Zustande die Eigensohaft hat, schweflige Säure und Sauerstoff zu verbinden) auf Bimsstein, gebranntem Ton oder andere ähnliche Substanz zu verteilen, zum Zweck, ein Gemisch von schwefliger Säure und Sauerstoff, atmosphärischer Luft oder andere brauchbare Substanz, welche Sauerstoff enthält, in Schwefelsäure umzuwandeln.

Im Jahre 1855 wird das englische Patent 183 an Ed. Schmersahl und Aug. Bouck ausgegeben und gibt an: ,Unsere Erfindung be- 
zieht sich auf die Darstellung von Schwefelsäure durch direkte Verbindung von Schwefligsäuregas mit Sauerstoff“. Um das auszuführen, erzeugen wir schweflige Säure durch Verbrennen von Schwefel oder durch andere Mittel und lassen sie im Gemisch mit Luft durch eine erhitzte oder erhitzte Röhren streichen. Um die Oberfläche zu vergrößern, bringen wir Asbest, ein Gemisch von Kupfer und Chromoxyd, Platin als Draht oder Blech, präparierten Koks, präparierten Bimsstein, tierische oder gewöhnliche Kohle, poröse Substanz, erzeugt durch Calcinieren von Ton mit organischer Substanz, oder andere poröse Substanzen in diese Röhren. Weiterhin wollen die Patentinhaber in das Gemisch von schwefliger Säure und Luft Dampf einblasen. Aus der früher schon zitierten Literatur ergibt sich, daß letzterer Gedanke allerdings recht unglücklich war, da zur Vereinigung von schwefliger Säure mit Sauerstoff nach dem Kontaktverfahren jede Beimengung von Wasserdampf äuBerst schädlich wirkt.

Es folgen die an $\mathrm{H}$ e $\mathrm{n} \mathrm{r}$ y $\mathrm{D}$ e a c o $\mathrm{n}$ im Jahre 1871 erteilten englischen Patente $\mathrm{Nr} .753$ und 1682. An Stelle der bisher vorgeschlagenen Oxyde unedler Metalle will Dea c o n Kupfersulfat als katalysierende Substanz verwenden. D e a c o $n$ beobachtet auch, daß die Reaktion besser verläuft, wenn man Luft oder Sauerstoff im Uberschuß verwendet.

Utber einige Einzelheiten macht der Erfinder folgende Angaben :

,Wenn man Schwefelsäure nach meiner Erfindung darstellt, so darf die Temperatur nicht den Punkt erreichen, bej welchem Kupfersulfat durch einen Strom von heißer Luft zersetzt wird, sonst kann nicht die ganze schweflige Säure in Schwefelsäure umgewandelt werden, denn Schwefelsäure selbst wird mehr oder weniger in schweflige Säure und Sauerstoff bei hoher Hitze zerlegt. Umgekehrt wird durch die Hitze allein schweflige Säure und Sanerstoff zum Teil zu Schwefelsäure vereinigt. DieAnwendung vonKupfersulfat oder einem anderen chemischen Reagens von ähnlicher Kraft bei irgend. einer gegebenen Temperatur bezweckt, die Bildung von Schwefelsäure zu vermehren und die Zersetzung zu vermindern, so daß bei irgend, einer gegebenen Temperatur durch die Anwendung von Kupfersulfat oder eines chemischen Aquivalents eine größere Menge schweflige Säure in Schwefelsäure umgewandelt wird, und $\mathrm{daB}$ durch dessen Gebrauch in einem richtigen Apparat und bei genügend tiefer Temperatur praktisch die ganze Menge Schwefelsäure umgewandelt wird". - Die beste Temperatur für die Darstellung von Schwefelsäure liegt unter dem Punkt, bei welchem Kupfersulfat in einem heißen Luftstrom zersetzt wird und über dem Schmelzpunkt des Zinns, und es ist zweekmäBig in großen Apparaten bei dieser angegebenen Temperatur und mit der gröBten praktischen $\mathrm{Zu}$ mischung von Luft (Sauerstoff) $\mathrm{zu}$ arbeiten.

Im Jahre 1875 publizierte C 1 e m e $n$ s W i n k le $r$ eine Arbeit, in welcher er die Einwirkung von platiniertem Asbest auf verschiedene Gemenge von schwefliger Säure mit Sauerstoff oder Luft untersuchte. Dabei glaubte er die Beobachtung $\mathrm{zu}$ machen, daß eine besonders weitgehende Vereinigung zu Sohwefelsäureanhydrid eintrat, sobald er mit reinem Sauerstoff arbeitete und diesem in richtigem stöchiometrischen Verhältnis mit schwefliger Säure über erhitzten platinierten Asbest leitete. Indem er diese Auffassung zur Grundlage seiner weiteren Versuche machte, suchte or auf billige Weise schweflige Säure und Sauerstoff ohne Beimengung anderer Gase im richtigen Mengenverhältnis zu erzeugen. $\mathrm{Zu}$ diesem Zwecke erschien ihm die Zersetzung konz. Schwefelsäure bei Glühhitze als günstigstes Darstellungsverfahren. Da die Schwefelsäure in schweflige Säure, Sauerstoff und Wasser zerfällt, braucht er nur durch Trocknen der Gase das Wasser zu entfernen, um dann gleich die Gase im Verhältnis zu haben, wie er sie zur Wiedervereinigung verwenden wollte.

Der Wert dieser $\mathrm{W}$ i $\mathrm{n} \mathrm{k}$ le $\mathrm{r}$ schen Publikation wurde allgemein überschätzt; denn seine Auffassung, daß schweflige Säure mit reinem Sauerstoff in stöchiometrischen Verhältnissen anzuwenden seien, um brauchbare Gasgemische zur Bildung von Schwefelsäureanhydrid zu bekommen, war unrichtig. Nach dem Guldberg W a a g e schen Gesetz ist die Verwendung eines Sauerstoffüberschusses von Vorteil, und dieser Anforderung waren schon frühere Arbeiten entgegengekommen, so z.B. Deacon in seinen oben erwähnten Patenten. Win k l e r s Annahme, daß Luftsauerstoff wegen seiner Verdünnung mit Stickstoff zur Ausführung des Kontaktverfahrens unbrauchbar sei, war ebenfalls nicht stichhaltig. Welterhin erwies sich die Herstellung des von W inkler gewünschten stöchiometrischen Gasgemisches aus konz. Schwefelsäure als technisch unbrauchbar. Gleichwohl wurde $W$ in $k$ le rs Verfahren vielerorts in den Großbetrieb einzuführen versucht. Die Entwicklung der organischen Farbstoffindustrie erforderte größere Quantitäten von Oleum zu Sulfurierungszwecken, als es bisher der Fall war, und deshalb beschäftigten sich 1877 folgende Werke (allerdings erfolglos) mit der Ausübung des Winkler schen Verfahrens. 1. Badische Anilin- und Soda-Fabrik. 2. Chem. Fabrik G. C. Zimmer in Mannheim. 3. Majert \& Co. in Schlebusch. 4. W. Squire und R. Messel in London. 5. Muldenhütten bei Freiburg i. S. Die Versuche auf den letztgenannten königl. Muldenhütten hatten nach einer Mitteilung in der deutschen Industrieztg. 1877, S. 417 zur Erkenntnis geführt, daß Arsen im Platinkontakt schädlich wirke, und es wurde daher zur Erzeugung mög. lichst arsenfreier Gase die zu zersetzende Schwefelsäure erst mit Schwefelwasserstoff behandelt, und so das Arsen ausgefällt. Diese Angabe ist namentlich deshalb interessant, weil ein Gehalt an Arsen, wie es scheint, lange Zeit der Ausführung des Kontaktverfahrens in der Badischen Anilin- und Soda-Fabrik Schwierigkeiten bereitet hat. Es sind denn auch im letzten Jahrzehnt auf die Entfernung von Arsen aus den Kontaktgasen eine Reihe von Patenten entnommen worden. Patentierbar kann aber, nach obiger Veröffentlichung, nicht das Prinzip, Arsen zu entfernen, sondern nur die Methode, wie es zu geschehen hat, sein.

Ahnlich, wie es schon in dem englischen Patent 731 vom Jahre 1853 vorgeschlagen wurde, verwenden $K$ on r. L ang e und K a r l Göpner nach dem französischen Patent 123906 vom Jahre 
1878 wiederum direkt Pyritröstgase, welche durch Pyritabbrände oder durch Platinschwamm in rotglühendem Zustande geleitet werden, und absorbieren das gebildete Schwefelsäureanhydrid nach erfolgter Kühlung durch mit konz. Schwefelsäure berieselte Türme zu rauchender Schwefelsäure.

Mit diesen Angaben mag die ältere Literatur über die Darstellung von Sehwefelsäure nach dem Kontaktverfahren beschlossen werden. Es käme eine Reihe neuerer Veröffentlichungen. Wiewohl die ältere Literatur in der neuesten Auflage von $\mathrm{L} u \mathrm{ng}$ es vorzüglichem Handbuch der Sodaindustrie auch eingehende Berücksichtigung gefunden hat, so erschien es doch nicht überflüssig, in einer Zeitschrift alte Veröffentlichungen einmal in einer Weise zusammenzustellen, daß derjenige, welcher die heutige Darstellung von rauchender Schwefelsäure kennt, sich bewußt wird, daß eigentlich längst Bekanntes heute modern ist, und daß die heute laufenden $\mathrm{Pa}$ tente zur Konstruktion einer Kontaktschwefelsäureanlage nicht absolut nötig sind.

\section{Zur Schwefelbestimmung im Pyrit.}

\section{Von G. Lunge. \\ (Eingeg. 2.j10. 1905.)}

Dennstedt und $\mathrm{HaBler}$ (diese Z. 18, 1562) glauben, die Ursache der Abweichungen in den Resultaten der Schwefelbestimmung in dem ,internationalen Pyritmuster" darin gefunden zu haben, daß bei der Aufnahme des Abdampfungsrückstandes nach der Aufschließung, wenn diese nach meiner Vorschrift „,mit 1 ccm konz. Salzsäure und $100 \mathrm{ccm}$ Wasser" geschehe, leicht basisches Ferrisulfat zurückbleibe. Dies werde vermieden, wenn man zuerst mit dem $1 \mathrm{ccm}$ konz. Salzsäure erhitzte und dann erst die $100 \mathrm{ccm}$ Wasser hinzusetze.

In bezug auf das letztere stimme ich mit den genannten Herren vollständig überein; ja noch mehr, seit den über 30 Jahren, daß ich meine Methode zur Schwefelbestimmung ausgearbeitet habe, $h$ a be ich selbstnie anders verfahren, und habe es auch meine schüler so ge le hrt. In meiner Vorschrift steht ja auch nicht, man solle $100 \mathrm{ccm} 0,3 \%$ iger Salzsäure anwenden, sondern $1 \mathrm{ccm} \mathrm{kon} \mathrm{z}$. Salzsäure und $100 \mathrm{ccm}$ Wasser, und das ist eben wie oben $\mathrm{zu}$ verstehen, nämlich daß man e rst die Salzsäure und d a n $n$ das Wasser zusetzt; ohnehin arbeitet man dabei immer auf dem Wasserbade und wird eben die konz. Salzsäure erst einige Minuten einwirken lassen, ehe man das Wasser zusetzt. Ich gebe zu, daB allenfalls der eine oder der andere mißverständlich die Salzsäure mit dem Wasser schon vor dem Zusatze verdünnen könnte, aber die meisten Chemiker müssen meine Vorsebrift doch richtig verstanden haben, denn sonst müßte bei den vielen Causenden von Bestimmungen nach meiner Methode obiger Fehler schon längst bemerkt und gerügt worden sein. Selbst ein ganz wenig geübter Chemiker kann doch den ,,zarten gelben Rückstand" von basischem Ferrisulfat nicht mit unaufgeschlossener Gangart verwechseln, und wenn ihm einmal ein derartiger Rückstand aufgestoßen ist, so wird er von selbst darauf kommen, daß man meine Vorschrift in der richtigen Weise auf. fassen müsse, die von den Herren De $n \mathrm{~ns} t \mathrm{edt}$ und $\mathrm{H}$ a $\beta \mathrm{l}$ er für neu gehalten wird.

Der beste Beleg dafür, daß die Abweichungen in den Resultaten für das internationale Pyritmuster nicht auf dem von den Herren $D$ e $n \mathrm{n}$ s ted $t$ und $\mathrm{H}$ a Bler angenommenen Grunde beruhen, ist folgender: Wenn jener Grund der richtige wäre, so hätten diejenigen Chemiker, welche, wie $\mathrm{D}$ e $\mathrm{n} \mathrm{n}$ s te d t und $\mathrm{H}$ a $B \mathrm{l}$ e $\mathrm{r}$, den Rückstand zuerst mit der konz. Salzsäure erwärmen, die $\mathrm{h} \ddot{o} \mathrm{~h}$ e r e n, die anderen aber die $\mathrm{n}$ i ed ri g e r e $\mathrm{n}$ Resultate gefunden. Nun gehören aber gerade die in $\mathrm{m}$ e i $\mathrm{n}$ e $\mathrm{m}$ Laboratorium gefundenen Resultate zu den niedrigsten, und doch kann ich positiv versichern, daß wir i $m$ e $r$ nach derselben Weise wie $D$ en $n$ s t e $d t$ und $H$ a $B l$ e r verfahren haben, und ebenso positiv, daß wir jenes basische Ferrosulfat $\mathrm{n}$ i c h t übersehen haben.

Die Ursache der erwähnten Differenzen hat sich inzwischen als ganz wo anders liegend herausgestellt, nämlich in der Art der Fällung mit Chlorbaryum, ob allmählich oder in einem Gusse. Diese Aufklärung verdanken wir den Herren Prof. $\mathrm{H}$ i $\mathrm{n} \mathrm{t} \mathrm{z}$ und Dr. W e b e $\mathbf{r}$ in Wiesbaden, welche in nächster Zeit in der "Zeitschrift für analytische Chemie“ darüber berichten werden. Infolge deren freundlicher brieflicher Mitteilung konnte auch ich mit Herrn $\mathrm{S}$ ti e r li n diesen Gegenstand bearbeiten; wir haben ganz dasselbe gefunden und werden ebenfalls an geeigneter Stelle darüber berichten. Für heute will ich nur sagen, daB nunmehr jeder Grund für eine größere Abweichung als $\dashv 0,1 \%$ Schwefel im Pyrit nach meiner Methode fortfällt.

Was die von den Herren Dennstedt und $\mathrm{H}$ a $\mathrm{B}$ l e r ausgearbeitete Bestimmung des nutzbaren Schwefels durch Verbrennung im Sauerstoffstrome betrifft, so erscheint sie mir durchaus rationell, und ich bezweifle ihre Genauigkeit um so weniger, als sie ja mit meiner richtig angewendeten Methode durchaus übereinstimmt. Es ist mir aber doch fraglich, ob sich die neue Methode zur allgemeinen Anwendung und zur Verdrängung der meinigen im Pyrithandel eignet, da sie immerhin erheblich mebr Apparatur und Zeit als die letztere erfordert und eben doch dieselben Resultate ergibt, wie ihre Erfinder selbst angeben.

$$
\mathrm{Z} \ddot{\mathrm{ur}} \mathrm{c} \mathrm{h}, \mathrm{l} \text {. Oktober } 1905 .
$$

\section{Glycerinbestimmungsmethode nach Dr. Shukoff und Dr. Schestakoff.}

Mitteilung aus dem Laboratorium der Dynamitfabrik Schlebusch.

(Eingeg. d. 16./8. 1905.)

Zur Naebprüfung der Methode von Dr. S h u k off und Dr. Schestak off haben wir eine Reihe Versuche angestellt und geben in nachstehendem die erhaltenen Resultate.

Die von Dr. Shuk off und Dr. Schesta$\mathrm{k}$ of $\mathrm{f}$ ausgearbeitete Methode oder, wie wir sie kurz nennen wollen, die Extraktionsmethode, hat ohne Zweifel gewisse Vorzüge, die ihre Anwendung sehr empfehlen. Sie ist zunächst eine direkte Me- 